\title{
Reduction of Gingival Black Triangles with Dental Pulp or Gingival Stem Cell Injection Therapy: Clinical and Outcomes Research Gaps
}

\author{
Jennifer Hilario Diaz ${ }^{1}$ and Jordana Kate Schmier ${ }^{2 *}$ \\ ${ }^{1}$ Diplomate, American Board of Periodontology and International Congress of Oral Implantologists, USA \\ ${ }^{2}$ Senior Managing Scientist, Exponent, USA \\ *Corresponding author: Jordana Schmier, Senior Managing Scientist, Exponent, 1800 Diagonal Road, Suite 500, Alexandria, \\ VA 22314, USA, Tel: 571-227-7241, E-mail: jschmier@exponent.com
}

\begin{abstract}
Gingival Black Triangles (GBTs), or gingival embrasures, are aesthetically displeasing even when they are not severe enough to require periodontal treatment. Multiple treatment modalities exist, including veneers, composite and other prosthetic modalities, with few, if any, treatments covered by insurance. Demand for GBT treatment is likely to increase with the aging of Baby Boomers, whose use of cosmetic dental procedures exceeds their predecessors. In recent days, stem cell therapy has gained more attention in the regeneration of oral and maxillofacial structures. We propose that the use of Dental Pulp Stem Cells (DPSC) or gingival stem cells for GBT treatment may have clinical, economic, and patient preference advantages that can fill a treatment gap, literally and figuratively. Safety and effectiveness concerns will persist until additional research is funded and conducted but the local nature of treatment would seem to expose patients to fewer risks than systemic use. Targeting the GBTs directly minimizes the long-term self-cleansing problem that other alternatives can create. Longevity is anticipated to exceed veneers and other prosthetic solutions. The cost of stem cell treatment for GBTs will likely be lower than veneers, consistent with the targeted placement. Further, less time in treatment and quicker recovery, both of which are anticipated, are likely to be preferable to patients. We enumerate these potential advantages and suggest a clinical way forward to evaluate the use of stem cell therapy for GBT treatment. Based on the anticipated differences for stem cell treatment of GBTs compared with usual care, we propose approaches for quantifying benefits from the patient perspective and from an economic standpoint. Future research will be needed to confirm the appropriateness of the recommended humanistic and economic evaluations.
\end{abstract}

\section{Keywords}

Adoption, Esthetics dental, Periodontics, Stem cells

\begin{abstract}
Abbreviations
DPSC: Dental Pulp Pluripotent Stem Cell; GBT: Gingival Black Triangles; QALY: Quality-Adjusted Life Year; QOL: Quality of Life; WTP: Willingness to Pay
\end{abstract}

\section{Introduction}

Cosmetic dentistry has increased in demand in recent years, with revenues in the US expected to grow from $\$ 16$ billion in 2015 to almost $\$ 28$ billion by 2024 [1]. These services include everything from over-the-counter bleaching products to removable appliances to veneers to implants. More than one-quarter of respondents in a survey of cosmetic dentists reported that patients initiate conversations about treatment options [1], which suggest many patients are preparing and researching prior to visits. Cosmetic dentists report that $88 \%$ of their patients consider cost a primary concern, with the only greater concern their appearance [1]. Many of these procedures are not or are only minimally covered by insurance, and the willingness to pay among consumers is constantly being tested in the real world. Dentists reported that more than $20 \%$ of their patients underwent more than $\$ 5,000$ worth of procedures in a year [1]. Almost three-fourths of cosmetic dentists reported offering a third-party financing service; most felt that it helped with patients' decision to have treatment [1]. Taken together, this means that there is a growing interest in cosmetic dentistry but that potential patients remain sensitive to costs.

Gingival Black Triangles (GBTs), or open gingival embrasures, rank highly among esthetic defects and are 
generally considered to be unsightly and prematurely age the smile [2]. They predispose interproximal areas to accumulate food debris and excessive plaque formation. Surgical and non-surgical management of GBTs may include, but is not limited to, preserving or reconstructing the affected soft tissue including the interdental papilla and alveolar bone; restorative techniques (including the use of veneer prostheses and gingival-colored ceramics); orthodontic treatment; or regeneration using tissue engineering and volumizers. Each of these approaches has clinical, economic, and patient preference advantages and disadvantages.

In this paper, we suggest a novel approach to reduce the appearance of gingival black triangles with the use of stem cell therapy, either injectable Dental Pulp Stem Cells (DPSC) or gingival stem cells, and discuss advantages and challenges from clinical, economic, and patient preference perspectives. With the increased focus on patient-centered research in the past decade, it is becoming more important to determine how to assess the patient perspective earlier in the clinical research process. Researchers can shorten the path to a full understanding of the economic and patient-reported outcomes and those findings can complement rather than trail behind clinical outcomes if they are included in studies even while potential clinical benefits are still being evaluated.

\section{Clinical Background}

Mesenchymal stromal or stem cells are phenotypically heterogenous populations of adult progenitors, initially isolated from the stroma of bone marrow by Friedenstein and coworkers [3]. Studies have identified that dental pulp can contain and be used as a source stem cells. Their embryonic origin, from neural crests, explains their multipotency. DPSCs can differentiate into different kinds of cells and tissues [4], especially bone-like tissue, and their multipotency has been compared to those of Bone Marrow Stem Cells (BMSCs). It has been demonstrated that proliferation, availability, and cell number of DPSCs are greater than BMSCs [5]. DPSC has also been used to regenerate nerves [6], cornea [7], bladder and renal tissues [8], skeletal muscles [9], lung tissue [10] and has demonstrated good angiogenic [11] and neurogenic potential.

Currently, the available strategies to treat black triangles are to either augment the papilla itself or graft autogenous tissue surgically. However, these available techniques have not been able to demonstrate predictable results. Yamada et al. investigated the potential of a tissue-engineered method for soft tissue augmentation with Mesenchymal Stem Cells (MSC), Platelet-Rich Plasma (PRP) and Hyaluronic Acid (HA) as the scaffold and achieved predictable results with aesthetic improvements in the black triangle [12]. This technique could emerge as a novel option for periodontal regenerative therapy in the near future [12].
Many animal studies have been conducted to regenerate periodontal tissues, bone, dentin and pulp tissues. Few human studies have been done to date. Although clinically, researchers have been able to regenerate periodontal-like tissue, bone and pulp-like tissue, they still have not been able to regenerate tissues that completely resemble tissues in their natural form. Ongoing research on periodontal regeneration therapies has explored issues as complex as tooth and bone development; GBT treatment is technically simpler. The future of stem cell therapy in dental applications looks promising. Concurrently, to further aim for an enhanced treatment outcome of cell therapy in dental applications, factors like scaffolding that can potentially influence the surrounding microenvironment by affecting the regional specification of the implanted stem cell should be investigated. A recent study by Ishihara and colleagues mentioned that nonphysiological scaffolds might disturb the physiologic circulation of the signaling molecules such as growth factors, which might influence cellular differentiation [13].

The application of this treatment with scaffold-free Cell Sheet Engineering (CSE), which is a new technology to regenerate injured or damaged tissues, should be considered. Cell sheet engineering uses a thermosensitive surface to form a dense cell sheet that can be detached when temperature decrease and can then be stacked on top of one another according to the thickness of cell sheet for the specific tissue regeneration application. This technique should provide excellent microenvironment for vascularization, since an intact cell matrix will be maintained for angiogenesis [14]. In addition, a study has shown that mesenchymal stem cells that were cultured as cell sheets and delivered as a scaffold-free cell sheet injection results in bone formation [15]. This hard tissue formation can be considered a positive contributory factor to assist in reducing the distance of contact point from the crest of the bone, thereby increasing the chance to have an interdental papillary fill [16]. Ongoing research and development of newer scaffolds, understanding various signaling molecules and their cues, understanding gene expression and proteomics of stem cells are the future directions that will take us a step forward to achieving successful regeneration.

\section{Clinical Benefits and Challenges}

There are multiple anticipated clinical advantages to DPSC or gingival stem cell treatment compared to other modalities but also questions about safety and efficacy that must be resolved.

Stem cell-based therapies bring with them new safety challenges. A particular difficulty is the ability to monitor cell biodistribution, since once administered, the cells may be essentially indistinguishable from host cells. The ability to track the therapeutic cells is key to an objective assessment of risk. But since GBT treatment would be administered locally, rather than intravenous- 
Iy, a lesser dissemination is likely to occur. However, the ability to determine the biodistribution of administered cells still raises technical issues, as monitoring the fate of exogenous cells will require the development of novel technologies. The detection of misplaced cells may necessitate a mechanism for their removal, which again may not be technically feasible at present. There is a need for technological advances in biomonitoring alongside the development of novel means for eliminating administered cells that become inappropriately located. Eliminating errant cells is likely to be a more challenging task and may involve incorporation of a "self-destruct" mechanism programmed into the cells to elicit apoptosis in response to a given stimulus. In order to minimize patient risk, each stage of the cell therapy production should be assessed for potential safety concerns before introduction to a human subject. This evaluation includes the manufacturing process itself, as well as the characterization and formal safety assessment of the finished product.

There are, however, several notable areas in which effectiveness could be superior to existing modalities. For example, careful positioning of veneers or crowns is important to avoid problems with bite or decay around the edges, a concern not applicable with stem cell treatment. Restorations for implants may not be as successful as teeth [17], whereas no such concern is relevant for GBT treatment with stem cells. Further, stem cell treatment may be appropriate for a broader patient population; for example, patients who grind their teeth are not good candidates for porcelain veneers or implants yet stem cell treatment would not be contraindicated.

The longevity of stem cell treatment of embrasures is unknown. However, if the problem re-emerges, the patient would have the option of multiple treatment modalities for the specific site. Once a crown or veneer is placed, though, the patient must replace it or risk tooth sensitivity and development of carious lesions (cavities). Ironically, although initial application of veneers for cosmetic reasons is rarely covered by insurance, replacement of damaged or lost veneers may be covered.

Evidence to support the use of stem cell therapy for this purpose, while available, remains limited [18-21]. Many small studies and case series have been published but as the technology and application are new, there is not yet evidence confirming the long-term persistence of the procedure. Increased research funding could facilitate the clinical research needed to confirm the optimism about the role of stem cells.

\section{Economic Evaluation}

In the past ten years, there were no papers indexed in PubMed (the National Library of Medicine's database of primarily peer-reviewed journals) that reported on costs of periodontal regenerative care in the United States. As such, suggesting a path for understanding the relative costs associated with treatment of GBT using various modalities is an exercise in estimation. However, this is not unlike other industries; pharmaceutical and device manufacturers consistently propose prices in a changing and evolving market. In the case of GBT treatment, in which costs would be set partly by practitioners and are likely borne by the consumer, the consumer's willingness to pay for one modality of treatment over another is a key element in the comparison of costs.

The gold standard for comparing costs of treatments is the cost-effectiveness analysis, [22] but the usual framework takes the perspective of an insurer (either commercial or government payer) and uses a common effectiveness measure such as the Quality-Adjusted Life Year (QALY) [22]. Patients rather than insurers would be responsible for most, if not all, costs for aesthetic treatment, so this approach must be modified for GBT cost assessment. Further, this so-called "third-party payer perspective" usually considers only direct medical costs in evaluating cost-effectiveness. There are no savings in medical costs associated with treating GBTs, unlike how treatment of severe persistent asthma with inhaled medication has been shown to decrease costs associated with exacerbations and hospital care. A cost comparison of stem cell treatment versus other techniques would likely favor stem cell treatment because veneers routinely require more visits and professional attention and typically cover multiple teeth. The insurer might have a vested interest in the success of treatments for GBTs, though, as the failure of crowns or veneers, even if initially covered by the patient, would likely become the insurer's responsibility to replace. There may be cases in which periodontal procedures reduce healthcare costs for other conditions [23]. In these situations, the insurer may have a particular interest; advocates could make the case that insurers should be less frugal about paying for periodontal regenerative treatment if it is likely to reduce costs for common chronic conditions. A traditional cost-effectiveness analysis, from the perspective of a third-party payer, may be useful to understand this scenario.

Less commonly, cost-effectiveness studies take a societal perspective, in which they also consider indirect costs, such as the cost associated with changes in mortality or work productivity, or the patient perspective, in which they consider out of pocket costs. In terms of indirect costs, there is anecdotal support that patients who undergo cosmetic procedures expect or report improvements in their perception of work productivity and income, similar to studies that suggest that better dental health may be associated with higher earnings in certain populations [24]. Web sites describing procedures explain how a "smile makeover" can improve self-confidence and help with job interviews and in-person sales and customer service careers. The extent to which income and career success, and thus indirect costs, may be objectively affected by GBT treatment remains un- 
known. These types of indirect costs may be similar across treatment modalities for GBT in the long term, aside from different rates of lost income or productivity associated with the initial visits and recovery.

Another type of economic evaluation is the Willingness to Pay (WTP) assessment, which seeks to understand the maximum price at which a consumer would purchase a good, in this case, GBT treatment. Comparing WTP for stem cell treatments to other modalities is difficult because the options differ on so many attributes, but since consumers rather than insurers would be paying, it may be more important than a traditional cost-effectiveness analysis. Understanding the array of attributes that would be compared is essential to developing a WTP study. Attributes would likely include cost, effectiveness of the treatment, duration of the treatment, time required to implement, and quality of life or satisfaction. Focus groups and semi-structured interviews with candidate patients could help identify which attributes and help establish the levels (descriptions) of each attribute.

Longevity is relevant to both cost-effectiveness and WTP assessments. Longevity ranges widely for alternative treatment modalities by material and care, from as little as five years for composite material to as long as 15-20 years for porcelain. The longevity of stem cell treatment for GBT has yet to be determined; costs are likely to be lower, on average, than other restorative procedures based on fewer visits and chair time. An economic model, however, should extend long enough to include eventual replacement of veneers or failure of stem cell treatment, particularly as younger patients are being treated.

At this point, there have been no economic comparisons of cosmetic dental procedures. This is not surprising, given their status as non-reimbursed services. However, patients' and practitioners' decision-making could benefit from an understanding of long-term costs associated with various treatment modalities. While there remain sources of uncertainty and improvements in technologies limit our confidence in assumptions, patients should have available predictions of costs associated with initial treatment and follow-up or revision care for GBT treatment before initiating treatment. To ensure that there is a cost story to tell, even early clinical studies should capture information that can help inform health economic modeling and identify evidence to support the value proposition for stem cell treatment of GBTs.

\section{Patient Preference and Quality of Life Advan- tages}

Successful reduction or elimination of GBTs is associated with patient satisfaction [25] but little is known about which treatment modalities are preferred by patients. Multiple factors can affect patient satisfaction, including pain, recovery time and lost productivity. Note that characteristics of treatment modalities like recovery time and lost productivity figure into both cost and patient preference analyses.

The number of visits required to complete treatment is determined by the number of teeth and areas affected. Veneers, for example, typically require a thorough case analysis with photographs, mounted study models and evaluation of occlusal scheme prior to initiating the actual preparation of teeth with anesthesia; temporary veneers may be applied. At this stage of treatment, the patient may be on her third visit with the treating restorative dentist. Prior to delivery and cementation of the veneers, another two or three short visits may take place to finalize the shape, shade and occlusal relation with opposing teeth. Follow-up visits will then take place upon delivery and cementation of veneers with frequency depending on the esthetic demand of the patient. In contrast, treatment of GBTs with stem cells is anticipated to require a single encounter following the initial consultation and planning visit. Follow-up care is likely to be substantially more time-consuming for veneers than for stem cell treatment, resulting in more "chair time" for the patient.

Longevity can be influenced by preparation technique and the condition of the tooth or teeth [26]. Again, this characteristic can be relevant to the cost evaluation as well as patient preferences. For patients who are employed, the multiple visits likely impinge on productivity. Also, transportation costs, delays in return to usual activities and lost productivity for general dental care have been shown to be substantial $[27,28]$. While patients choosing to undergo cosmetic procedures have already implicitly indicated that they have disposable income and/or time, less time away from work and usual activities and quicker recovery are undoubtedly preferred.

It is understood that serious deficiencies in oral and periodontal health is associated with decrements in patient quality of life (QOL) [20,29-32]. Cost-effectiveness studies often use QOL metrics as measures of effectiveness. It is unknown whether GBTs are associated with decrements in QOL. Studies of QOL associated with dental, orthodontic, and periodontal needs often focus on psychosocial well-being [33-35], but since QOL includes other dimensions that are unlikely to be affected by improvements to esthetics, notably physical functioning, it is unclear whether commonly-used measures of QOL would be able to capture improvements associated with treatment and therefore be meaningful assessment tools. Unlike more severe conditions, GBTs are unlikely to be associated with pain or difficulty eating, and probably have little to no impact on physical functioning. Prospective studies on stem cell treatment should include patient-centered evaluations, such as QOL and satisfaction, in order to include this information into decision-making and treatment selection. 


\section{Addressing Information Gaps}

There are substantial information gaps in terms of the clinical utility of stem cells for GBT treatment and the patient-reported and economic outcomes of their use. In terms of clinical knowledge, there is not yet a definitive study that has explored the feasibility, safety and effectiveness of the approach. Scaffold-free cell sheet engineering and successfully managing biodistribution of cells present opportunities and challenges. While these areas are explored, however, we suggest that even early human studies can include metrics that would help identify economic and humanistic outcomes, both of which are particularly important for a treatment in which patients are the primary consumers rather than insurers. We have identified key elements of a prospective pilot study on patient reported measures, including baseline measurements of the GBT and self-reported oral QOL prior to prophylaxis preceding the stem cell injection. Pain assessment, using a scale that could be compared to one or more existing studies of other restorative techniques, would be measured during and after the procedure. Follow-up assessments would include clinical and QOL evaluations, along with documentation by the clinician of all health care resources used and surveying patients about satisfaction and time lost from work or usual activities. If possible, interviews or focus groups with patients who are considering various treatments and with those who have completed treatment could contribute to identifying other evaluations to include. Thoughtful analysis of these data could provide a starting point for larger prospective studies that could build our understanding of what appears to be a viable and minimally invasive alternative to an increasingly-prevalent problem.

\section{Conclusions}

The potential for stem cell for gingival embrasure treatment is enormous, as other treatment modalities place a significant time and cost burden on patients. In clinical situations when there are concerns other than GBTs, more invasive or complex procedures may be advisable. Continued research should help to address questions about success, longevity and possible contraindications. With the number of patients with GBTs increasing and demand for cosmetic procedures growing, it will become important to assist patients with evaluating treatment options. As the costs and benefits are more likely to be realized directly by patients rather than insurers, a more complete understanding of these topics will be valuable to informing patient decision-making and, taking a step back, help develop a health economic and outcomes story for continued research. Particularly for a treatment in which the consumer is the patient rather than an insurer, conducting research into patient priorities alongside clinical research is important to adoption of a new procedure or technology.

\section{References}

1. American Academy of Cosmetic Dentistry (2015) Cosmetic dentistry state of the industry survey 2015.

2. Singh VP, Uppoor AS, Nayak DG, Shah D (2013) Black triangle dilemma and its management in esthetic dentistry. Dent Res J (Isfahan) 10: 296-301.

3. Friedenstein AJ, Chailakhyan RK, Latsinik NV, Panasyuk AF, Keiliss-Borok IV (1974) Stromal cells responsible for transferring the microenvironment of the hemopoietic tissues. Cloning in vitro and retransplantation in vivo. Transplantation 17: 331-340.

4. Khanna-Jain R, Vanhatupa S, Vuorinen A, Sandor GKB, Suuronen R, et al. (2012) Growth and differentiation of human dental pulp stem cells maintained in fetal bovine serum, human serum and serum-free/xeno-free culture media. J Stem Cell Res Ther 2: 126.

5. Alge DL, Zhou D, Adams LL, Wyss BK, Shadday MD, et al. (2010) Donor-matched comparison of dental pulp stem cells and bone marrow-derived mesenchymal stem cells in a rat model. J Tissue Eng Regen Med 4: 73-81.

6. Hei WH, Kim S, Park JC, Seo YK, Kim SM, et al. (2016) Schwann-like cells differentiated from human dental pulp stem cells combined with a pulsed electromagnetic field can improve peripheral nerve regeneration. Bioelectromagnetics.

7. Syed-Picard FN, Du Y, Lathrop KL, Mann MM, Funderburgh ML, et al. (2015) Dental pulp stem cells: a new cellular resource for corneal stromal regeneration. Stem Cells Transl Med 4: 276-285.

8. Hirose $Y$, Yamamoto T, Nakashima M, Funahashi Y, Matsukawa $Y$, et al. (2016) Injection of dental pulp stem cells promotes healing of damaged bladder tissue in a rat model of chemically induced cystitis. Cell Transplant 25: 425-436.

9. Li D, Deng T, Li H, Li Y (2015) MiR-143 and miR-135 inhibitors treatment induces skeletal myogenic differentiation of human adult dental pulp stem cells. Arch Oral Biol 60: 1613-1617.

10. Wakayama H, Hashimoto N, Matsushita Y, Matsubara K, Yamamoto N, et al. (2015) Factors secreted from dental pulp stem cells show multifaceted benefits for treating acute lung injury in mice. Cytotherapy 17: 1119-1129.

11. Janebodin $\mathrm{K}$, Zeng $\mathrm{Y}$, Buranaphatthana $\mathrm{W}$, leronimakis $\mathrm{N}$, Reyes M (2013) VEGFR2-dependent angiogenic capacity of pericyte-like dental pulp stem cells. J Dent Res 92: 524531.

12. Yamada $Y$, Nakamura S, Ueda M, Ito K (2015) Papilla regeneration by injectable stem cell therapy with regenerative medicine: long-term clinical prognosis. J Tissue Eng Regen Med 9: 305-309.

13. Ishihara K, Nakayama K, Akieda S, Matsuda S, Iwamoto Y (2014) Simultaneous regeneration of full-thickness cartilage and subchondral bone defects in vivo using a three-dimensional scaffold-free autologous construct derived from high-density bone marrow-derived mesenchymal stem cells. J Orthop Surg Res 9: 98.

14. Moschouris K, Firoozi N, Kang Y (2016) The application of cell sheet engineering in the vascularization of tissue regeneration. Regen Med 11: 559-570.

15. Akahane M, Shigematsu H, Tadokoro M, Ueha T, Matsumoto $T$, et al. (2010) Scaffold-free cell sheet injection results in bone formation. J Tissue Eng Regen Med 4: 404-411. 
16. Tarnow DP, Magner AW, Fletcher $P$ (1992) The effect of the distance from the contact point to the crest of bone on the presence or absence of the interproximal dental papilla. $J$ Periodontol 63: 995-996.

17. Cosyn J, Raes M, Packet M, Cleymaet R, De Bruyn H (2013) Disparity in embrasure fill and papilla height between toothand implant-borne fixed restorations in the anterior maxilla: a cross-sectional study. J Clin Periodontol 40: 728-733.

18. Han J, Menicanin D, Gronthos S, Bartold PM (2014) Stem cells, tissue engineering and periodontal regeneration. Aust Dent J 59: 117-130.

19. Kim SH, Seo BM, Choung PH, Lee YM (2010) Adult stem cell therapy for periodontal disease. Int J Stem Cells 3: 1621.

20. Inglehart MR (2015) Enhancing periodontal health through regenerative approaches: a commentary on the need for patient-reported outcomes. J Periodontol 86: S4-S7.

21. Tassi SA, Sergio NZ, Misawa MY, Villar CC (2017) Efficacy of stem cells on periodontal regeneration: Systematic review of pre-clinical studies. J Periodontal Res.

22. Sanders GD, Neumann PJ, Basu A, Brock DW, Feeny D, et al. (2016) Recommendations for conduct, methodological practices, and reporting of cost-effectiveness analyses: second Panel on Cost-Effectiveness in Health and Medicine. JAMA 316: 1093-1103.

23. Jeffcoat MK, Jeffcoat RL, Gladowski PA, Bramson JB, Blum JJ (2014) Impact of periodontal therapy on general health: evidence from insurance data for five systemic conditions. Am J Prev Med 47: 166-174.

24. Glied S, Neidell M (2008) The economic value of teeth Working Paper 13879, in NBER Working Paper Series. National Bureau of Economic Research: Cambridge, MA.

25. Awartani FA, Tatakis DN (2016) Interdental papilla loss: treatment by hyaluronic acid gel injection: a case series. Clin Oral Investig 20: 1775-1780.
26. Shetty A, Kaiwar A, Shubhashini N, Ashwini P, Naveen D, et al. (2011) Survival rates of porcelain laminate restoration based on different incisal preparation designs: An analysis. J Conserv Dent 14: 10-15.

27. Harford J, Chrisopoulos S (2012) Productivity losses from dental problems. Aust Dent J 57: 393-397.

28. Hayes A, Azarpazhooh A, Dempster L, Ravaghi V, Quinonez C (2013) Time loss due to dental problems and treatment in the Canadian population: analysis of a nationwide cross-sectional survey. BMC Oral Health 13: 17.

29. Buset SL, Walter C, Friedmann A, Weiger R, Borgnakke WS, et al. (2016) Are periodontal diseases really silent? A systematic review of their effect on quality of life. J Clin Periodontol 43: 333-344.

30. Ferreira MC, Dias-Pereira AC, Branco-de-Almeida LS, Martins CC, Paiva SM (2017) Impact of periodontal disease on quality of life: a systematic review. J Periodontal Res 52: 651-665.

31. Haag DG, Peres KG, Balasubramanian M, Brennan DS (2017) Oral conditions and health-related quality of life: a systematic review. J Dent Res 96: 864-874.

32. Franke M, Broseler F, Tietmann C (2015) Patient-related evaluation after systematic periodontal therapy - a clinical study on periodontal health-related quality of life (PHQoL). Oral Health Prev Dent 13: 163-168.

33. Dumitrescu AL, Toma C, Lascu V (2009) Self-liking, self-competence, body investment and perfectionism: associations with oral health status and oral-health-related behaviours. Oral Health Prev Dent 7: 191-200.

34. Solomon D, Katz RV, Bush AC, Farley VK, McGerr TJ, et al. (2016) Psychosocial impact of anterior dental esthetics on periodontal health, dental caries, and oral hygiene practices in young adults. Gen Dent 64: 44-50.

35. Oland J, Jensen J, Elklit A, Melsen B (2011) Motives for surgical-orthodontic treatment and effect of treatment on psychosocial well-being and satisfaction: a prospective study of 118 patients. J Oral Maxillofac Surg 69: 104-113. 